\title{
Adverse reactions to piperacillin in adults with cystic fibrosis
}

\author{
RJ STEAD, HG KENNEDY, ME HODSON, JC BATTEN \\ From the Cardiothoracic Institute, Brompton Hospital, London
}

\begin{abstract}
Nine adult patients with cystic fibrosis, nearly a quarter of the 38 patients with this disease who were treated with piperacillin ( 59 courses in all) during 1981-3 at the Brompton Hospital, developed a swinging pyrexia after a mean of 13.5 days' treatment with this antibiotic. The fever resolved shortly after the piperacillin treatment was stopped, as did the widespread rashes in the two patients who developed them. Three of four patients who had probable reactions to azlocillin may have been sensitised by piperacillin. As piperacillin does not appear to be any more effective than other antipseudomonal penicillins in cystic fibrosis, it is no longer used at the hospital for treating bronchopulmonary exacerbations in such patients.
\end{abstract}

Chronic bronchopulmonary infection is the main cause of morbidity and mortality in cystic fibrosis. In most adults this is associated with the persistent presence of Pseudomonas aeruginosa in cultures of sputum. Piperacillin sodium is a semisynthetic ureidopenicillin with high in vitro activity against $P s$ aeruginosa.' ${ }^{\prime}$ We have recently noted unexpected fever in nearly a quarter of our patients with cystic fibrosis treated with piperacillin, ${ }^{2}$ and we report details of our experience with piperacillin in comparison with carbenicillin and azlocillin.

\section{The patients}

Thirty-eight patients with cystic fibrosis ( 24 men, 14 women; mean age 22.6 years) admitted because of deterioration in their bronchopulmonary condition were treated with 59 courses of piperacillin from 1981 to 1983 . All patients received $4 \mathrm{~g}$ of piperacillin six hourly as an intravenous infusion given over 20 minutes. The premixed $4 \mathrm{~g}$ vials were stored at room temperature and dissolved in sterile water immediately before administration. In addition, an aminoglycoside was administered by bolus intravenous injection eight hourly. Patients with pathogens other than Ps aeruginosa in sputum cultures also received appropriate oral antibiotics.

Address for reprint requests: Dr RJ Stead, Cardiothoracic Institute, Brompton Hospital, London SW3 6HP.

Accepted 24 September 1984

\section{Results}

Nine patients (24\%) developed swinging pyrexia of around $38.5^{\circ} \mathrm{C}$ after a mean of 13.5 days' treatment (range 5-28 days), having been afebrile earlier in their period in hospital. In all cases the fever resolved shortly after discontinuation of piperacillin and usually within 24 hours. Two patients developed widespread rashes, in one purpuric and in the other maculopapular, which faded soon after piperacillin had been discontinued. One of these patients also complained of low back pain at the time of piperacillin infusion and was found to have proteinuria. A patient with similar back pain had normal urine, and neither patient had other abnormalities of renal function. No patient had either lymphadenopathy or arthralgia. Two of the nine patients were given a subsequent course of piperacillin before the cause of the pyrexia was recognised and both developed further fevers.

The nine patients who had reactions were all male ( $p<0.01$, Fisher's exact test) and tended to have had longer courses of piperacillin. The reactions did not appear to be dose (table) or batch related. Three patients were receiving prednisolone for their pulmonary condition in doses of at least $15 \mathrm{mg} /$ day at the time of the reaction.

For comparison, the records of patients who received carbenicillin or azlocillin in combination with an aminoglycoside were reviewed. Thirty five patients received 47 courses of carbenicillin from 1981 to 1983 . Two $(6 \%)$ developed urticarial reactions immediately after carbenicillin infusions; both 
Details of 59 courses of piperacillin given to 38 patients with cystic fibrosis (values are means with standard deviations in parentheses)

\begin{tabular}{|c|c|c|c|}
\hline & $\begin{array}{l}\text { Course length } \\
\text { (days) }\end{array}$ & $\begin{array}{l}\text { Daily dose } \\
\text { (mg/kg body weight) }\end{array}$ & $\begin{array}{l}\text { Cumulative dose } \\
\text { (g/kg body weight) }\end{array}$ \\
\hline $\begin{array}{l}\text { Reactions to piperacillin } \\
n_{1}=9 \\
n_{2}=13\end{array}$ & $13.5(6.8)^{*}$ & $379(111)$ & $7.31(5.70)^{*}$ \\
\hline $\begin{array}{l}\text { No reaction to piperacillin } \\
n_{1}=29 \\
n_{2}=46\end{array}$ & $10.5(2.6)$ & $366(73)$ & $6.02(3.27)$ \\
\hline Student's unpaired $t$ test & NS & NS & NS \\
\hline
\end{tabular}

${ }^{*}$ Taken up to the onset of the reaction. $n_{1}$-number of patients; $n_{2}$-number of treatment courses; NS - not statistically significant.

had previously received multiple courses of carbenicillin. Thirty six patients received 43 courses of azlocillin during $1979-83$. Four patients $(11 \%)$ had probable reactions to azlocillin; three of these developed fever and two maculopapular rashes, which settled within 48 hours of discontinuation of azlocillin. It was noteworthy that three of the patients who reacted to azlocillin had previously been exposed to piperacillin ( $p<0.025$, Fisher's exact test), although only one had reacted to the latter. Two of these patients had received uneventful courses of azlocillin before their first course of piperacillin. On the other hand, there was no relationship between prior exposure to azlocillin and reactions to piperacillin.

\section{Discussion}

Low grade fever is common in patients with cystic fibrosis during bronchopulmonary exacerbations, but it rapidly resolves with antibiotic treatment. Swinging pyrexia later in a treatment course, however, is very unusual and there was no doubt clinically that it was related to piperacillin. Winston $\mathrm{et}^{a^{3}}$ used piperacillin in 59 patients with various serious disorders. Twenty per cent developed "definite or probable" reactions which were relatively mild and reversible, consisting mainly of eosinophilia and diarrhoea, and three patients had "drug fever". Reactions appeared to be related to longer treatment courses. In a study of 55 patients ${ }^{4}$ with cystic fibrosis seven of 20 patients treated with azlocillin and 14 of 30 treated with piperacillin developed fever towards the end of a 14 day course. Sixteen of the 21 patients also developed a generalised rash. Six patients noticed back pain with infusions, although it was not associated with abnormalities of renal function and, as in our cases, the aetiology was not clear. Twenty of the patients treated with azlocillin or piperacillin had, however, previously shown carbenicillin "allergy" or "intolerance," presumably related to repeated courses. In another unit (JL Blumer, personal communication) $30 \%$ of 200 patients with cystic fibrosis treated with piperacillin developed reactions similar to those we have described after 10-12 days' treatment, and Agostini et $a^{5}$ noted onset of fever at this time in $18.7 \%$ of 32 courses of piperacillin given to patients with cystic fibrosis. Lastly, Strandvik reported adverse reactions in 13 out of 18 patients with cystic fibrosis treated with piperacillin. ${ }^{6}$

Our clinical impression was that reactions to other penicillins had not been frequent in our patients with cystic fibrosis and this was supported by review of patients' records; the high incidence of reactions with piperacillin is therefore of concern. Furthermore, the incidence of reactions to azlocillin after treatment with piperacillin suggests that piperacillin may sensitise patients to other penicillins. The aetiology of the reactions and the observation that they were confined to men are unexplained; they did not appear to be dose related and were not prevented by concurrent corticosteroid treatment. Further immunological studies are needed, particularly to look for development of penicillin antibodies. Penicillin skin tests were not performed on our patients and systemic provocation tests were not considered ethically acceptable. Our clinical impression is that piperacillin does not hold any advantage in efficacy over other antipseudomonal penicillins in this group of patients and we have therefore discontinued its use in patients with cystic fibrosis.

We would like to thank Miss M Rehahn for statistical advice. RJS is supported by the Frances and Augustus Newman Foundation.

\section{References}

1 Wise R, Andrews JM, Bedford KA. Comparison of the in vitro activity of Bay k 4999 and piperacillin, two new antipseudomonal broad-spectrum penicillins, with other beta-lactam drugs. Antimicrob Agents Chemother 1978; 14:549-52.

2 Stead RJ, Kennedy HG, Hodson ME, Batten JC. Adverse reactions to piperacillin in cystic fibrosis. Lancet 1984;i:857-8.

3 Winston DJ, Murphy W, Young LS, Hewitt WL. Piperacillin therapy for serious bacterial infections. $A m J$ Med 1980;69:255-61. 
4 Moller NE, Eriksen KR, Feddersen D, et al. Chemotherapy against Pseudomonas aeruginosa in cystic fibrosis. A study of carbenicillin, azlocillin or piperacillin in combination with tobramycin. Eur J Respir Dis 1982;63:130-9.

5 Agostini M, Barlocco G, Bonomi U, et al. Alternative antibiotics against pseudomonas infections in cystic fibrosis. In vitro activity, pharmacokinetics, and double-blind randomized clinical trial with azlocillin, piperacillin, cefoperazone, ceftazidime, cefsulodin, cefotaxime and moxalactam. Preliminary results. Drugs in Experimental and Clinical Research 1983;9:671-86.

6 Strandvik B. Adverse reactions to piperacillin in patients with cystic fibrosis. Lancet 1984;i: 1362.

\section{Book notices}

Computed Tomography of the Chest. J David Godwin. (Pp 492; £59-25.) Lippincott. 1984.

A reference book of computed tomography of the chest is something most thoracic physicians and surgeons now need on their bookshelf. This book will fill the void well for the time being and, in a field that is changing rapidly as the machines improve and experience in different diseases accumulates, that is no mean feat. The book is well produced with illustrations on virtually every page and each CT scan clearly captioned. The introductory chapters on normal anatomy and technical aspects provide a clear and well illustrated foundation for the following chapters, which deal systematically with the entire thoracic contents and their diseases and abnormalities. The index is comprehensive and accurate and each chapter ends with a realistic summary of the "state of the art" and a useful list of references. Any textbook is likely to be incomplete or even out of date when the subject covered is advancing, but despite this many physicians and surgeons, and not a few radiologists, will find this book to be an essential addition to their library.-DCTW.

Tuberculosis. Praeger Monographs in Infectious Disease. Vol 2. David Schlossberg. (Pp 361; £37.75.) Praeger Publishers. 1983.

This volume is a "state of the art" statement of the American view of tuberculosis, produced in part to celebrate the centenary of the discovery of the tubercle bacillus. After a relatively brief introductory section considering epidemiology, pathogenesis, bacteriology, diagnosis, treatment, and prophylaxis, there are sections on the manifestations of tuberculosis in both its common and uncom mon sites in the body. There is a final relatively brief section on non-tuberculous mycobacterial infection. For those working in the field the volume is disappointing. It is not a comprehensive review of the subject. It presents few new insights and its level is relatively superficial. The balance of the section on clinical manifestations is odd; problems of the lungs are dealt with in 15 pages of text while the same amount of space is allocated to upper respiratory tuberculosis and seven pages are given to ocular tuberculosis. Tuberculous lymphadenitis, which is described as being "now seen infrequently," is dealt with in a section of 17 pages, while bone disease is allocated 19. In many of these sections one is left with the strong impression that the decline in incidence in tuberculosis in America has been so great that the authors are speaking from a position of theoretical rather than practical knowledge. The approach concentrates very heavily on the American problems in diagnosis and management. Little of the advice appears to be unusual or unsound but when we turn to problem areas in diagnosis and management insights and additional information are uncommon. It is difficult to see that this book has a place for the European physician. It is not orientated towards his practice or his problems, neither is it the sort of fundamental work which would be part of a reference library on tuberculosis.-MW McN

Clinical Manifestations of Respiratory Disease. Terry $\mathbf{R}$ Des Jardins (Pp 433; \$32.95.) Year Book Medical Publishers Inc. 1984.

This book, aimed mainly at nurses, students, and physiotherapists, has been written by a respiratory therapist with the aim of bringing together the physiology, anatomy, aetiology, clinical pathophysiology, and treatment of respiratory disease into one comprehensive volume. Sadly, this laudable aim is not achieved. The section on anatomy and pathophysiology is straight forward and generally accurate, although the analogy of water flow through garden sprinklers for airflow is overused. The sections on aetiology are thin, with no mention of bronchial hyperreactivity and only passing reference to the role of cigarette smoke. Given the aim of correlating pathology and physical signs, it is surprising that fibrosing alveolitis and pleural effusion are not mentioned at all in view of their classical physical signs. But the clinical aspects of this book are very weak-lung sounds are described archaically and incorrect statements abound (for example, clubbing is common in advanced emphysema). The sections on management are brief and inaccurate: ephedrine is stated at one point to be a xanthine drug and percussion physiotherapy to be useful in acute asthma and as an optional treatment for pulmonary oedema, and it is said that "alcohol may be aeriosolised into the patient's lungs to lower the surface of the frothy secretions." These examples are not isolated, and although the artistic quality of the diagrams are good the book is misleading and confusing and cannot be recommended.-JGA 